\title{
Physical activity as a determinant of bone conservation in the radial diaphysis in rheumatoid arthritis
}

\author{
D Shawe, R Hesp, J M Gumpel, P N Sambrook, J Reeve
}

\begin{abstract}
Objectives-To determine if increasing physical activity is protective of diaphysial (cortical) bone mass

Methods-Fifteen patients attending two rheumatology clinics who had developed seropositive or classical rheumatoid arthritis up to 26 months previously were studied prospectively for two to three years. Rates of loss (or gain) in bone mass in the radial diaphysis and the trabecular bone of the distal radius were measured by quantitative computed tomography, and in the spine by dual photon absorptiometry. Physical activity was assessed by the Framingham physical activity index. Disease activity was followed at threemonthly clinic visits at which the haemoglobin, erythrocyte sedimentation rate, and platelet count were measured. The urinary hydroxyproline to creatinine ratio and plasma osteocalcin were measured at the beginning and end of the observation period.
\end{abstract}

Results-Eleven patients required treatment with disease modifying drugs but none was given corticosteroids. Those whose physical activity did not improve lost radial diaphysial bone at about $4 \%$ annually. There was, however, a statistically significant inverse relation, accounting for $48 \cdot 5 \%$ of the variance, between bone loss at this site and improvement in physical activity as assessed by the Framingham index. The other two sites showed much weaker associations. Adjusting for indices of disease activity hardly affected the first relation. Three biochemical indices related to bone turnover showed weak tendencies to decrease with increasing physical activity.

Northwick Park

Hospital and MRC

Clinical Research

Centre,

Harrow,

United Kingdom

D Shawe

R Hesp

J M Gumpel

P N Sambrook

J Reeve

Correspondence to: Dr J Reeve,

University Department of Medicine,

Addenbrooke's Hospital, Cambridge CB2 2QQ United Kingdom.

Accepted for publication 23 March 1993
Conclusions-Peripheral cortical bone, distant from inflamed joints, is conserved more successfully in patients who achieve higher levels of physical rehabilitation. This may have implications for avoiding long bone fractures later in the disease.

(Ann Rheum Dis 1993; 52: 579-581)

Generalised bone loss occurs in established rheumatoid arthritis (RA); suggested causative factors include immobility, disease effects, reduced adrenal androgens, and possibly treatment with corticosteroids. ${ }^{1}$ Periarticular osteoporosis develops early and may relate to the release of cytokines and other inflammatory mediators in the affected joint. ${ }^{2}$ The causes of generalised osteoporosis in RA are more controversial. $^{34}$ In a cross sectional study, Sambrook et $a l^{4}$ found that physical activity, measured with the Framingham physical activity index (PAI), ${ }^{5}$ was an independent predictor of femoral neck bone mineral density, along with age and body weight. The relation was weak $(p<0.05)$, however, perhaps owing to the cross sectional nature of the study.

We have therefore examined prospectively whether the PAI is a predictor of bone conservation in three sites: the spine, the distal radius, and the radial diaphysis. At these sites the dispersion of bone loss rates was greater in a group of patients with early $\mathrm{RA}$ than in controls (variance ratios $F$; for spine $F=2 \cdot 61$, $p=0.05$; for radial diaphysis $F=3 \cdot 36$, $\mathrm{p}<0.025),{ }^{6}$ suggesting that some factor associated with RA and its evolution was causing individual bone density trends to diverge more than is seen normally.

\section{Patients and methods}

Fifteen of 16 women recruited by Sambrook et $a l^{6}$ to a prospective study of predictors of bone loss in early RA were available for follow up after two to three years, and agreed to repeat assessments of the PAI. The table gives their clinical details. At the same time the biochemical markers of bone formation and resorption (plasma osteocalcin (Incstar, Stillwater, MA, USA) and urinary fasting calcium to creatinine and hydroxyproline to creatinine ratios) were remeasured. Each patient had been followed up at three monthly intervals in the intervening period with routine measurements of haemoglobin, erythrocyte sedimentation rate (ESR; Westergren), and platelet count. Four had not yet reached the menopause. Clinical management was aimed at reducing joint inflammation and destruction and no patient was treated with systemic corticosteroids. All but four patients required disease modifying drugs and all received nonsteroidal anti-inflammatory drugs, as previously described. ${ }^{6}$ Throughout the follow up period, bone densitometry was performed at

\section{Clinical details of patients at the start of the study}

Mean (range) age (years)

No of postmenopausal wome $57 \cdot 8(36-67)$

Mean (range) 10 $\begin{array}{ll}\text { Mean (range) time since the menopause (years) } & 10 \cdot 2(2-22) \\ \text { Mean (range) disease duration (months) } & 15 \cdot 6(5-27)\end{array}$ 
three to four month intervals for a mean of $2 \cdot 3$ years. Measurements were made by computed tomography ${ }^{7}$ of the trabecular bone at the distal radius ( $5 \mathrm{~cm}$ from the joint surface) and of cortical bone at the diaphysial midshaft (one third of the distance from the ulnar styloid to the olecranon), and in L2-L4 by dual photon absorptiometry (Novo BMC Lab 22a). Coefficients of variation for individual measurements were estimated as 2,2 , and $2 \cdot 6 \%$ respectively. As we clearly showed that rates of loss at the distal trabecular site were non-linear over time,${ }^{6}$ bone loss at this site was estimated as the difference between the first and last measurements and are reported as the yearly loss. The other rates were determined as the regression coefficients in linear regression analyses against time because they appeared to be linear. All three loss rates were compared with the change in the PAI by linear regression analyses.

Because of the possibility that the observed correlation between PAI (or PAI changes) and diaphysial bone mass changes could be confounded by an effect of disease activity, results are presented with and without adjustment for mean levels of the three markers of disease activity that were regularly measured-that is, ESR, haemoglobin concentration, and platelet count. Significance levels for the two bone measurement sites distant from active disease were adjusted by a factor of two because two sites were measured (Bonferroni correction).

\section{Results}

All but four patients showed some improvement in their PAI and the mean value increased significantly (initial mean (SD) PAI $30.1(2.6)$, mean improvement 3.53 (5.58); $\mathrm{p}=0.03)$. The four patients not requiring disease modifying drugs did relatively better in this respect (mean improvement $+9.6 v+0.7$ in those requiring disease modifying drugs; $\mathrm{p}<0.01)$. The change in PAI was strongly and inversely related to diaphysial bone loss in the radius (figure), the regression shown accounting for $48.5 \%$ of the variance in diaphysial bone loss. A similar relation was seen in the 11 patients receiving disease modifying drugs when considered separately. Much less of the variance in radial trabecular or lumbar spinal bone loss rates was accounted for $(10.0$ and $1.1 \%$ respectively; $p>0.05)$. When the final value of the PAI over the time of observation was used as the independent variable, the results were similar (the percentages of variance accounted for were $54 \cdot 7,9 \cdot 4$, and $6 \cdot 6 \%$ respectively)

The final measurements of the three indices relating to bone turnover, the plasma osteocalcin, the urinary hydroxyproline to creatinine ratio, and the urinary calcium to creatinine ratio were correlated with the concurrent PAI; the PAI was found to explain $10.7,28.8$, and $21.8 \%$ of the variance in these indices, suggesting in the case of hydroxyproline $(p<0.05$ unadjusted) an inverse relation between physical activity and

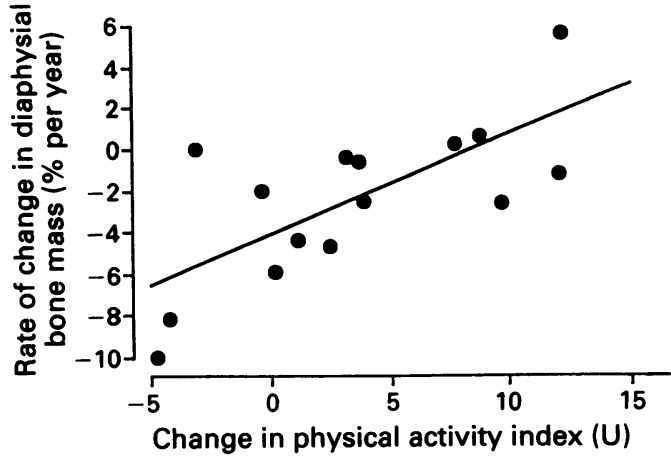

Annual percentage change in radial diaphysial bone mass compared with change in Framingham activity index. The regression equation is $y=0 \cdot 47 x-4 \cdot 17$ (Student's $\mathrm{t}=3 \cdot 50$ for regression coefficient; $p=0.004$ or $p=0.008$ with Bonferroni correction; $r=0 \cdot 70$ ). For the 11 patients receiving disease modifying drugs, this equation is $y=0.69 x-4.94$ (Student's $\mathrm{t}=4 \cdot 89 ; p<0 \cdot 002$ )

bone resorption two to three years after recruitment to the study.

The mean rate of diaphysial bone loss showed some correlation with the mean ESR $(\mathrm{r}=-0 \cdot 42 ; \mathrm{p}=0 \cdot 1)$, suggesting a weak relation between sustained disease activity and loss of diaphysial bone. When the relation between rate of diaphysial bone loss with the change in PAI was adjusted in turn for mean levels of the three indices of disease activity (ESR, platelet count, and haemoglobin concentration), however, the strength of the relation between physical activity and bone conservation was largely unaffected (regression coefficient for simple regression $0.047 \quad(0.014)$; adjusted coefficients, $0.058(0.020), 0.072(0.015)$, and $0.071(0.015)$ respectively; all values of $\mathrm{p} \leqslant 0.024$ with the Bonferroni correction).

There was no significant difference in mean bone loss rates at any of the three sites between those patients who were and were not treated with disease modifying drugs ( $p>0 \cdot 05)$, nor did diaphysial bone loss rates correlate with age or years since the menopause $(p>0.05)$. The postmenopausal group lost $1 \cdot 6 \%$ more diaphysial bone than the premenopausal women, but this was not significant $(p>0.05)$ Adjusting the correlations of bone loss with physical activity for age or menopausal status had little effect.

\section{Discussion}

These observations show an impressive relation between recovering physical activity in response to treatment of $\mathrm{RA}$ and protection against further diaphysial (cortical) bone loss from the radius. The inverse relation between the use of disease modifying drugs and the improvement of the PAI presumably reflects the use of these drugs in those patients whose disease was more severe. Like Sambrook et al in their cross sectional study, ${ }^{4}$ we could not confirm an independent effect of physical activity on spinal bone loss. Near inflamed joints other factors appear to be more important. We showed previously that bone loss from the distal radius, adjacent to the inflamed wrist joint, was related to plasma levels of parathyroid hormone and calcitriol. ${ }^{6}$ 
As the worker (PNS) who initially assessed these patients had returned to Australia, it was not possible to compare formally the results obtained by the two PAI assessors. As all the first round of PAI values were obtained by PNS and all the second round by DS, any bias that may have arisen is likely to have been consistent. We compared the calculated bone density trends with final PAI values (depending on the results of only one assessor) as well as with changes in the PAI, and the conclusions drawn were similar. The Framingham index is comparatively simple, easily understood, and robust. For some purposes it may be thought insensitive, but a broad range of values was found in these patients after two to three years of observation.

The biochemical investigations suggest that in those patients who are less active bone resorption is increased, whereas physical activity may prevent bone loss by suppressing bone resorption. It is possible that the different responses of the radius and spine reflect a more substantial effect of active disease on inhibiting the mechanical use of the forearm compared with the spine. Verstraeten and Dequeker ${ }^{3}$ showed that in patients with RA not treated with corticosteroids the radial diaphysial bone mass was significantly reduced compared with controls. Als $e t a l^{9}$ found, in a cross sectional study, that reductions of forearm bone mineral content were associated with the degree of functional impairment and that biochemical markers of bone remodelling such as plasma osteocalcin (formation) and urinary hydroxyproline (resorption) increased as function was impaired. ${ }^{9}$ Alternatively, the increased urinary hydroxyproline in those less active may reflect disease activity as urinary hydroxyproline is also derived from the complement component Clq, the turnover of which is also increased in RA. ${ }^{10}$ In another study, Sambrook et $a l^{11}$ found that hip and spine bone loss responded differently to exogenous oestrogen treatment in postmenopausal women with RA, with no apparent protection being conferred at the hip. These findings suggest the mechanisms of spinal bone loss in postmenopausal women with RA may be different from those responsible for bone loss in long bones.
In our previous study based on these patients, ${ }^{6}$ we found that loss of trabecular bone from the distal radius about $2.5 \mathrm{~cm}$ from the inflamed wrist joint was much more rapid than from other sites and was statistically positively associated with plasma parathyroid hormone and calcitriol levels. It seemed possible that these circulating hormones might interact biochemically with local mediators of increased bone resorption, such as interleukin 1, to amplify juxta-articular bone loss in the vascular territory of the wrist joint. ${ }^{6}$

In conclusion, early disease control allowing the resumption of pre-disease levels of physical activity may be the most effective means of preventing bone loss from the cortices of the peripheral skeleton and might reduce the otherwise increased risk of disabling long bone fractures in later life. Other approaches will be required to control juxta-articular bone loss.

1 Reid D M, Kennedy N S J, Smith M A, Tothill P, Nuki G. Total body calcium in rheumatoid arthritis: effects of disease activity and corticosteroid treatment. BMF 1982; 285: $330-2$.

2 Sambrook P N, Reeve J. Bone disease in rheumatoid arthritis. Clin Sci 1988; 74: 225-30.

3 Verstraeten A, Dequeker J. Vertebral and peripheral bone mineral content and fracture incidence in postmenopausal patients with rheumatoid arthritis: effect of low dose corticosteroids. Ann Rheum Dis 1986; 45: 852-7.

4 Sambrook P N, Eisman J A, Champion G D, Yeates M G, Pocock N A, Eberl S. Determinants of axial bone loss in rheumatoid arthritis. Arthritis Rheum 1987; 30: $721-8$.

5 Kannel W B, Sorlie P. Some health benefits of physical activity. The Framingham study. Arch Intern Med 1979; 139: 857-61.

6 Sambrook P N, Shawe D, Hesp R, et al. Rapid periarticular bone loss in rheumatoid arthritis. Possible promotion by normal circulating concentrations of parathyroid hormone or calcitriol (1,25-dihydroxyvitamin $\left.\mathrm{D}_{3}\right)$. Arthritis Rheum 1990; 33: 615-22.

7 Elsasser U, Reeve J. Bone density measurements with computed tomography. Br Med Bull 1980; 35: 293-6.

8 Krolner B, Pors Nielsen S. Measurement of bone mineral content (BMC) of the lumbar spine. I. Theory and application of a two-dimensional dual photon attenuation method. Scand $\mathcal{F}$ Clin Lab Invest 1980; 40: 653-63.

9 Als O S, Gottfredson A, Riis B J, Christiansen C. Are disease duration and degree of functional impairment determinants of bone loss in rheumatoid arthritis? Ann Rheum Dis 1985; 44: 406-11.

10 Reid K B M, Lane D M, Porter R R. Isolation and characterisation of $\mathrm{C} 1 \mathrm{q}$, the sub-component of the first of complement from human rabbit sera. Biochem $\mathcal{F}$ 1972; 130: 749-63.

11 Sambrook P, Birmingham J, Champion D, et al. Postmenopausal bone loss in rheumatoid arthritis: effect of estrogens and androgens. $\mathcal{f}$ Rheumatol 1992; 19: $357-61$. 